\title{
REPORT
}

\section{Are you listening? Exploring a developmental knowledge-action dissociation in a speech interpretation task}

\section{J. Bruce Morton and Yuko Munakata}

\author{
Department of Psychology, University of Denver, USA
}

\begin{abstract}
Young children sometimes act inappropriately despite appearing to know what to do. Dissociations of this kind raise important questions about the organization and development of knowledge and action systems. The present study investigated a knowledgeaction dissociation in 6-year-olds performing a speech interpretation task and tested the hypothesis that knowledge-action dissociations stem from a general difficulty resolving conflicting cues. When knowledge and action measures were equated in terms of the amount of conflict that needed to be resolved for a correct response, children's knowledge no longer appeared to outstrip their ability to act appropriately. Implications of the findings for competing views of knowledge representation and knowledgeaction system organization are discussed.
\end{abstract}

\section{Introduction}

Young children sometimes act inappropriately despite appearing to know what to do. For example, when 3year-olds are asked to sort cards according to one set of rules, and then to switch and sort the same cards according to new rules, they will often continue to sort the cards according to the old rules despite verbally reporting the new rules (Zelazo, Frye \& Rapus, 1996). What might account for this apparent dissociation?

Children might possess the requisite knowledge for acting appropriately in these tasks, but err because of problems of inhibitory control (Carlson, Moses \& Hix, 1998; Gerstadt, Hong \& Diamond, 1994). In this view, knowledge and action systems are structurally independent, and children's correct answers to the simple knowledge questions suggest their knowledge is intact.

However, such apparent knowledge-action dissociations may instead reflect differences in the conditions under which knowledge and action are assessed (Munakata \& Yerys, 2001). For example, in the Dimensional Change Card Sort (DCCS; Zelazo \& Frye, 1997) described above, knowledge of the new rules is tested under non-conflicting conditions (e.g. 'In the shape game, where do the trucks go?'), whereas sorting is assessed under conditions of interference (i.e. test cards contain conflicting shape and color information). Thus, success in the knowledge test coupled with failure in the sorting test may be due in part to differences in the way knowledge and sorting are measured. In support of this idea, when the knowledge test contains the same conflicting information as the sorting test (e.g. 'Where do the red trucks go in the shape game?'), the systematic knowledge-action dissociation disappears (Munakata \& Yerys, 2001). Children who sort incorrectly but answer the standard knowledge questions correctly tend to fail the conflict questions. These findings suggest that apparent knowledge-action dissociations can obscure a general difficulty resolving conflict, and therefore raise questions about the structure and functional interrelatedness of knowledge and actions systems.

Recently, a striking knowledge-action dissociation has been observed in much older children performing a speech interpretation task, raising the possibility of extending this investigation to a new task and an older age group (Morton \& Trehub, 1999; Morton, Trehub \& Zelazo, in preparation). In the task, 6-year-olds are presented with utterances that contain conflicting cues to emotion (e.g. 'My dog ran away from home' spoken happily). Initially, children are asked to base their responses on what the speaker says (i.e. if she describes a happy situation, then respond 'happy', but if she describes a

Address for correspondence: J. Bruce Morton, Department of Psychology, University of Western Ontario, London, Ontario, Canada N6A 5CZ; e-mail: bmorton3@uwo.ca or Yuko Manakata, Department of Psychology, Campus Box 345, University of Colorado at Boulder, Boulder, CO 80309-0345, USA; e-mail: munakata@psych.colorado.edu 
sad situation, then respond 'sad'). After reaching a performance criterion, children are then instructed to switch and base their responses on the speaker's tone of voice (i.e. her paralanguage). Despite showing knowledge of the new paralanguage rules, many 6-year-olds persist in responding in terms of what the speaker says rather than how she speaks (Morton \& Trehub, 1999).

However, as in the DCCS, knowledge and action are tested under different conditions. Knowledge of the post-switch rules is tested under non-conflicting conditions by asking children 'If the speaker's voice sounds like this - hummed happy paralanguage - then what is the right answer in the new game? And if it sounds like this - hummed sad paralanguage - then what is the correct answer?' In contrast, children's ability to put these rules into action is tested under conditions of conflict (i.e. in the presence of competing propositional cues). Thus, the dissociation may be due in part to differences in the way knowledge and action are measured.

The purpose of the present experiment, then, was to test the hypothesis that what underlies this apparent knowledge-action dissociation is a general difficulty resolving conflicting cues (Munakata \& Yerys, 2001). We tested this hypothesis by instructing 6-year-olds to switch from content to paralanguage and then testing their knowledge of these instructions using both standard (i.e. non-conflicting) and conflict questions (e.g. 'If she says something happy and her voice sounds sad, then what is the correct answer?'). Poor switching and correct responses to conflict questions would provide stronger evidence of a knowledge-action dissociation. Poor switching and incorrect responses to conflict questions (with correct answers to non-conflict questions) would suggest a general difficulty resolving conflict.

\section{Method}

\section{Participants}

Twenty (12 males, 8 females) 6-year-olds (6;0 to 6;3) participated in the experiment. Participants were recruited through the University of Denver Developmental Participant Pool. Parents received $\$ 5$ as compensation for their time and travel, and children received a small gift.

\section{Apparatus and stimuli}

Test stimuli consisted of six utterances, three with happy content spoken in a sad tone of voice (e.g. 'My mommy gave me a treat' spoken sadly) and three with sad content spoken in a happy tone of voice (e.g. 'My dog ran away from home' spoken happily). Two additional utterances, one with happy content spoken in a happy tone of voice and one with sad content spoken in a sad tone of voice, were used as probe stimuli. All utterances were spoken by the same female speaker and were digitally recorded on a Radius Macintosh clone computer using SoundScope software. Previous testing (Morton \& Trehub, 2001) confirmed that both the content and the tone of voice in these materials are readily interpretable by 6 -year-old children. Materials were presented in a random order.

\section{Procedure}

Participants were tested individually on a computer in a quiet room. Children sat facing the computer and the experimenter sat beside them. The experimenter used the computer keyboard to call for trials, and children entered their responses using a button box. One button, labeled the 'happy button', had a drawing of a happy face on it, while the other button, labeled the 'sad button', had a drawing of a sad face on it. The procedure was run on a PC clone $466 \mathrm{MHz}$ computer using EPrime software, and consisted of three blocks of trials: a bias assessment, a pre-switch and a post-switch block.

The bias assessment block was presented to children as the first of three games and was designed to assess each child's initial preference for content or paralanguage. Children were told at the beginning of the first game that they would hear a friend of the experimenter's named Marianne, and that they were to listen to Marianne and decide whether she felt happy or sad. If they thought she felt happy, they were to press the happy button but if they thought she was sad, they were to press the sad button. Participants were presented with all six test utterances.

The pre-switch block followed the bias assessment, and was presented to children as the second game. Children were instructed to attend to the content of the utterances as follows: 'Listen to what Marianne says, and if she says something happy, then the correct answer is "happy" (the experimenter pointed to the happy button), but if she says something sad, then the correct answer is "sad" (the experimenter pointed to the sad button).' Multiple trials were administered until children reached a criterion of $80 \%$ correct over eight trials. Two of the six test utterances, one with happy content and sad paralanguage, and one with sad content and happy paralanguage, were used in this block. The same two utterances were used for each child, although the order of presentation was randomized for each participant. Children received feedback on their performance. For correct responses, the computer went 'bing' and the word 'good' appeared on the screen. For incorrect responses, the computer went 'boing' and a black ' $\mathrm{X}$ ' appeared on 
the screen. Upon reaching criterion, children heard a probe trial featuring an utterance with happy content and paralanguage. Participants who correctly attended to content in the pre-switch phase would respond 'happy' on the probe trial, whereas children who attended to paralanguage but generated correct responses by 'doing the opposite' would respond 'sad' on the probe trial. No children used this latter strategy to reach criterion.

The post-switch block was presented to children as the last game. Children were told that in the last game, they were not to listen to what Marianne was saying, but instead were to listen to the sound of her voice. To clarify what it meant to listen to someone's voice, and to ensure that children were able to label examples of affective paralanguage, the experimenter engaged each participant in a short dialogue about happy and sad voices. Children were asked how someone's voice sounds when they feel happy and how it sounds when they are sad. Children were then told that the experimenter was going to provide some examples of happy and sad voices, and that they had to determine whether the voice sounded happy or sad. The experimenter then hummed one example each of happy and sad paralanguage (i.e. a happy or sad voice with no words) and asked the child whether the voice sounded happy or sad. All children labeled both examples correctly. The rules were then reiterated, and the game began. The same two utterances that were used in the pre-switch block were used in the post-switch block. All participants received five test trials (three with happy content and sad paralanguage, and two with sad content and happy paralanguage) in the same random order. Children received no feedback on their performance. Following the five test trials, children heard a probe trial featuring sad content and paralanguage. Children who generated correct responses by attending to content but giving the opposite response would respond 'happy' to the probe trial. No child used this strategy.

Following the probe trial, children answered a series of questions. First, to ensure participants distinguished between listening to the speaker's voice and listening to what she said, children were asked to recall what they were listening to in the last game and what they were not listening to. Most children answered these questions correctly, with 18 children correctly responding that they were listening to the speaker's voice and 15 children correctly responding that they were not listening to what she was saying. Participants were then asked the standard and conflict knowledge questions. For the standard questions, children were asked 'If Marianne's voice sounds like this - hummed sad paralanguage what button will you press? And if her voice sounds like this - hummed happy paralanguage - what button will you press?' Children were asked one of two forms of the conflict question. The first was 'If Marianne says something (happy/sad), and her voice sounds (sad/happy), then what is the right answer in this game?' The second was 'If Marianne's voice sounds (happy/sad) and she says something (sad/happy), then what is the right answer in this game?' Each form had two versions, one in which 'happy' was the first term and sad the second, and the other in which 'sad' was the first term and 'happy' the second. Half of both the switchers (i.e. children who correctly switched from content to paralanguage) and non-switchers (i.e. children who persisted in responding to content) received one form of the conflict question, while the other half received the alternative form. Children's responses to the knowledge questions were recorded by the experimenter on a separate sheet of paper and entered into the computer after the entire procedure was completed.

\section{Results}

Performance in the six bias assessment trials indicated that most children came into the experiment showing a preference for content. Responses consistent with content received a score of 0 while responses consistent with paralanguage received a score of 1 . Thus, scores could range from 0 to 6 , with low scores indicating a preference for content and high scores indicating a preference for paralanguage. Because of a non-normal distribution, scores ranging from 0 to 2 and 4 to 6 were categorized as 'content-focused' and 'paralanguagefocused', respectively. In all, 17 scores were categorized as 'content-focused', and one was categorized as 'paralanguage-focused'. Two scores of 3 were dropped from this particular analysis because they reflected no preference for either content or paralanguage. Chi-square analysis confirmed that a significantly greater proportion of scores were 'content-focused' than one would expect by chance alone $\left(\chi^{2}(1, N=18)=10.8, p<0.01\right)$.

Pre-switch performance was also virtually identical across all participants. Seventeen children reached criterion without making a single error. Two children made one mistake, and one child made three mistakes. All children reached criterion and did so by attending to content, as confirmed by the probe trial.

Post-switch performance varied non-normally, with $75 \%$ of children correctly switching to paralanguage on every trial (6 of 20), or not switching at all (9 of 20). Thus, children were classified as either switching or not switching, where switching was defined as at least three out of five correct responses. Children were classified as passing the standard knowledge questions 
Table 1 Numbers of children $(\mathrm{N}=20)$ passing and failing standard and conflict questions, and switching to paralanguage in the post-switch block

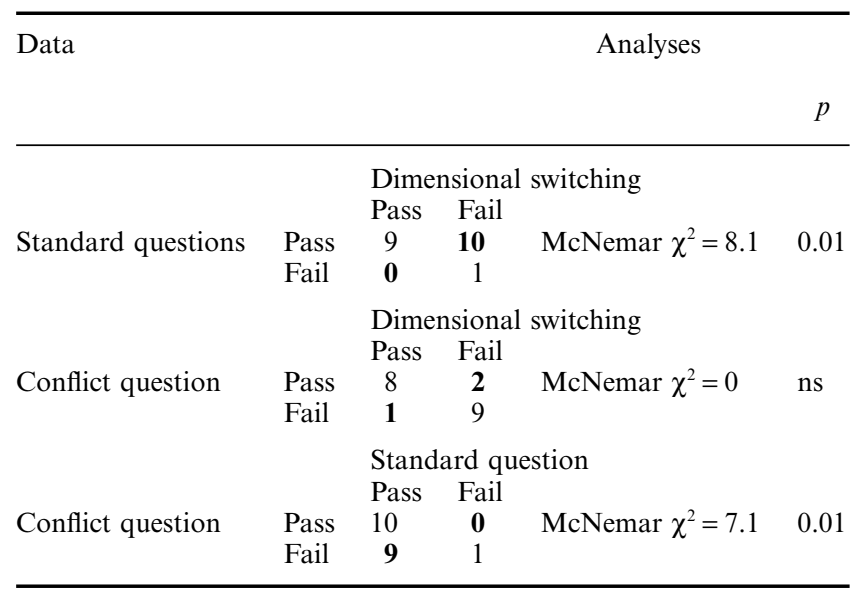

Note: Critical comparisons are in bold

if they answered both questions correctly. Due to nonnormal distributions, we used McNemar's $\chi^{2}$ (with Yates correction for small cell entries) to analyze the data, ${ }^{1}$ consistent with previous studies (Munakata \& Yerys, 2001).

Table 1 shows the classifications for the three comparisons of interest. First, many children remembered the post-switch rules as assessed by the standard questions, but failed to use them. Specifically, ten children passed the standard questions but failed to switch, whereas no child failed the standard questions and switched (McNemar's $\chi^{2}=8.1, p<0.01$ ). However, this systematic knowledge-action dissociation disappeared when the conflict question was used to assess knowledge. Most children either passed both measures $(N=8)$ or failed both measures $(N=9)$. The few remaining children were as likely to pass the conflict question and fail to switch as they were to fail the conflict question and pass at switching (McNemar's $\left.\chi^{2}=0, p=1.0\right){ }^{2}$ Finally, many children who answered the standard questions correctly failed the conflict question, whereas no children showed

\footnotetext{
${ }^{1}$ McNemar's $\chi 2$ allows us to test the dissociation of interest, whether children perform better on one measure than on another measure, in terms of whether the pattern of passing one measure while failing a second measure is more likely than the pattern of failing the first measure while passing the second measure. This test approximates a sign test, which Zelazo et al. (1996) used to assess the knowledge-action dissociation. The two analyses yielded the same results with the current study. We focus on the McNemar for simplicity.

${ }^{2}$ Following Zelazo et al. (1996), we also conducted this analysis using correlation, and obtained the same result. Switching and conflict question performance were positively and significantly associated $(\phi(20)=$ $0.7, p<0.002)$.
}

the reverse pattern (McNemar's $\chi^{2}=7.1, p<0.01$ ). An equal number of children passed each form of the conflict question, indicating that there was no difference in the difficulty of the two forms of the conflict question.

\section{Discussion}

Children's knowledge can at times appear to outstrip their ability to act on that knowledge (Gerstadt et al., 1994; Morton \& Trehub, 1999; Zelazo et al., 1996). However, these apparent dissociations may be due in part to differences in the way knowledge and action are typically measured (Munakata \& Yerys, 2001). Often, knowledge is tested under non-conflicting conditions (i.e. conditions that include only the currently relevant dimension), whereas action is tested under conflicting conditions (i.e. conditions that include both the currently and the previously relevant dimensions; Morton \& Trehub, 1999; Morton et al., in preparation; Zelazo et al., 1996). In the present study, we attempted to measure knowledge and action under more equivalent conditions of conflict, and the apparent knowledge-action dissociation disappeared. This parallels earlier results showing that children's apparent knowledge of new rules can falter when tested under conditions of conflict (Munakata \& Yerys, 2001).

Although conflict questions were more difficult for children than non-conflict questions, these differences may have been attributable to factors other than the presence or absence of conflict. Non-conflict questions were syntactically and logically simpler than conflict questions, and included concrete instances rather than abstract descriptions of the relevant stimulus features. We included these differences to avoid an overly simple non-conflict question and an overly cumbersome conflict question. Had children been asked a non-conflict question that had an abstract description of the paralanguage (e.g. 'If Marianne's voice sounds happy, then what is the right answer?'), children could have easily responded in terms of the description (i.e. 'happy') without considering the meaning of the question. Children could not use this strategy when responding to the conflict question, however, because the question contained both the correct and the incorrect answer (i.e. 'happy' and 'sad'). To ensure that children did not respond in terms of the most recent alternative, the order of the correct and incorrect alternatives was counterbalanced across children. Children were as likely to answer the question correctly when the correct alternative was the most recent (e.g. 'If Marianne says something happy and her voice sounds sad ... ?') as when the incorrect alternative was the most recent (e.g. 'If Marianne's voice sounds 
sad and she says something happy...??). Although it was possible to use concrete exemplars in the conflict question, this would have made the question extremely cumbersome and potentially uninterpretable (e.g. 'If Marianne says "My dog ran away from home" and her voice sounds like this - hummed happy paralanguage - then what is the right answer?'). Finally, previous findings indicate that when differences in the syntactic complexity of conflict and non-conflict questions are controlled for, the same pattern of findings is observed (Munakata \& Yerys, 2001). Indeed, children perseverate on content given the actual stimuli. This can be explained in terms of conflict, but not syntactic complexity. Thus, while it remains an important empirical question as to whether other factors contributed to differences in the difficulty of conflict and non-conflict questions in the current study, the collection of evidence suggests that these differences were related to the presence or absence of conflict.

The present findings argue against the view that children possess the requisite knowledge for succeeding in rule-use tasks so long as they can answer simple questions about the new rules (Gerstadt et al., 1994). Instead, children's knowledge appears somewhat superficial, and is clearly inadequate for dealing with instances of conflict. This raises the possibility that changes in how knowledge is represented rather than changes in inhibitory control (Dempster, 1993; Houde, 2000) may account for developmental advances in consciously controlled behavior.

One possibility is that resolving conflict requires reflection (Zelazo, 2000). When a happy situation is described with sad paralanguage, or an utterance is described as consisting of happy content and sad paralanguage, rules based on content and paralanguage specify opposite responses. Therefore, to act appropriately under conditions of conflict, children need to consider carefully which pair of rules to apply. In one view, this requires reflection and the formulation of a higher-order rule whose primary function is to allow the appropriate pair of rules to be selected under conditions of conflict (Zelazo, 2000).

Still, this interpretation assumes that knowledge and action systems are structurally and functionally independent. However, it may be more biologically plausible to view knowledge as graded in nature and embedded within interactive rather than separable knowledge-action systems (McClelland, 1993; Munakata, McClelland, Johnson \& Siegler, 1997; Thelen \& Smith, 1994). In this view, a range of behaviors are thought to reveal knowledge, but the degree of knowledge revealed by any single behavior will vary with the strength of the underlying representation. Thus, in the present study, children's knowledge of task-related rules may be strong enough to lead to correct responding under non-conflicting conditions, but insufficiently strong to lead to correct performance under conflicting conditions. We have implemented this account, as applied to the present speech interpretation task and to the DCCS, in a neural network simulation (Morton \& Munakata, 2002; Munakata, Morton \& Stedron, in press). A graded view of representation has also informed models of cognitive functioning in a variety of other domains (see Munakata, 2001 for a review), including infants' memory for hidden objects (Munakata et al., 1997; Munakata, 1998), adults' ability to regulate cognitive processing under conditions of interference (Cohen, Dunbar \& McClelland, 1990), and patients' ability to recognize objects (Sitton, Mozer \& Farah, 2000).

Outstanding challenges remain, however, for all accounts of the development of higher-order cognitive control. In particular, it is unclear why mechanisms proposed to operate and bring about control in one task do not appear to be operative in the context of another task much later in development. Structurally, the present task is very similar to the DCCS (Zelazo \& Frye, 1997) in that participants are required to remember simple rules and use them to initiate a switch from one dimension of a stimulus to another. However, the present task is mastered much later in development than the DCCS. If 4year-olds master the DCCS because they are able to reflect on their knowledge (Zelazo, 2000) or maintain strong, active representations (Morton \& Munakata, 2002; Munakata \& Yerys, 2001), why do 6-year-olds fail the present task? Perhaps children's strong bias to content (Friend, 2000; Friend \& Bryant, 2000; Morton \& Trehub, 2001) places additional demands on these mechanisms (Morton \& Munakata, 2002). Further research is required to understand the basis of this fascinating decalage.

\section{References}

Carlson, S.M., Moses, L.J., \& Hix, H.R. (1998). The role of inhibitory processes in young children's difficulties with deception and false belief. Child Development, 69 (3), $672-$ 691.

Cohen, J.D., Dunbar, K., \& McClelland, J.L. (1990). On the control of automatic processes: a parallel distributed processing model of the Stroop effect. Psychological Review, 97 (3), 332-361.

Dempster, F.N. (1993). Resistance to interference: developmental changes in a basic processing mechanism. In M.L. Howe \& R. Pasnak (Eds.), Emerging themes in cognitive development (Vol. 1, pp. 3-27). New York: Springer-Verlag.

Friend, M. (2000). Developmental changes in sensitivity to vocal paralanguage. Developmental Science, 3, 148-162. 
Friend, M., \& Bryant, J.B. (2000). A developmental lexical bias in the interpretation of discrepant messages. Merrill-Palmer Quarterly, 46, 342-369.

Gerstadt, C.L., Hong, Y.J., \& Diamond, A. (1994). The relationship between cognition and action: performance of children 3.5-7 years old on a Stroop-like day-night test. Cognition, 53, 129-153.

Houde, O. (2000). Inhibition and cognitive development: object, number, categorization, and reasoning. Cognitive Development, 15, 63-73.

McClelland, J.L. (1993). The GRAIN model: a framework for modeling the dynamics of information processing. In D.E. Meyer \& S. Kornblum (Eds.), Attention and performance $X I V$ : Synergies in experimental psychology, artificial intelligence, and cognitive neuroscience (pp. 655-688). Mahwah, NJ: Lawrence Erlbaum Associates.

Morton, J.B., \& Munakata, Y. (2002). Active versus latent representations: a neural network model of perseveration, dissociation, and decalage. Developmental Psychobiology, 40, 255-265.

Morton, J.B., \& Trehub, S.E. (1999). The prepotency of speech content for 6-year-olds. Poster presented at the first meeting of the Cognitive Development Society, Chapel Hill, NC, October.

Morton, J.B., \& Trehub, S.E. (2001). Children's understanding of emotion in speech. Child Development, 72, 834-843.

Morton, J.B., Trehub, S.E., \& Zelazo, P.D. (in preparation). The development of flexible attention to emotion cues in speech.

Munakata, Y. (1998). Infant perseveration and implications for object permanence theories: a PDP model of the A not B task. Developmental Science, 1, 161-184.

Munakata, Y. (2001). Graded representations in behavioral dissociations. Trends in Cognitive Sciences, 5 (7), 309-315.
Munakata, Y., McClelland, J.L., Johnson, M.J., \& Siegler, R.S. (1997). Rethinking infant knowledge: toward an adaptive process account of successes and failures in object permanence tasks. Psychological Review, 104, 686-713.

Munakata, Y., Morton, J.B., \& Stedron, J.M. (in press). The role of prefrontal cortex in perseveration: developmental and computational explorations. To appear in P. Quinlan (Ed.), Computational models of development.

Munakata, Y., \& Yerys, B.E. (2001). All together now: when dissociations between knowledge and action disappear. Psychological Science, 12 (4), 335-337.

Sitton, M., Mozer, M.C., \& Farah, M.J. (2000). Superadditive effects of multiple lesions in a connectionist architecture: implications for the neuropsychology of optic aphasia. Psychological Review, 107 (4), 709-734.

Thelen, E., \& Smith, L.B. (1994). A dynamic systems approach to the development of cognition and action. Cambridge, MA: MIT Press.

Zelazo, P.D. (2000). Self-reflection and the development of consciously controlled processing. In P. Mitchell \& K.J. Riggs (Eds.), Children's reasoning and the mind (pp. 169189). Hove, UK: Psychology Press.

Zelazo, P.D., \& Frye, D. (1997). Cognitive complexity and control: a theory of the development of deliberate reasoning and intentional action. In M. Stamenov (Ed.), Language structure, discourse, and the access to consciousness (pp. 113-153). Amsterdam: John Benjamins.

Zelazo, P.D., Frye, D., \& Rapus, T. (1996). An age-related dissociation between knowing rules and using them. Cognitive Development, 11, 37-63.

Received: 12 April 2001

Accepted: 9 October 2001 